\title{
IMU-RGBD Camera 3D Pose Estimation and Extrinsic Calibration: Observability Analysis and Consistency Improvement
}

\author{
Chao X. Guo and Stergios I. Roumeliotis
}

\begin{abstract}
In this paper, we address the problem of extrinsically calibrating an inertial measurement unit (IMU) with respect to an RGBD sensor. In particular, we study the observability of the nonlinear IMU-RGBD calibration system and prove that the calibration parameters are observable given observations to a single point feature. Moreover, we show that the system has four unobservable directions corresponding to the global translation and rotations about the gravity vector. Based on the results of the observability analysis, we design a consistency-improved, observability constrained (OC) extended Kalman filter (EKF)-based estimator for calibrating the sensor pair while at the same time tracking its pose and creating a 3D map of the environment. Finally, we validate the key findings of the observability analysis and assess the performance of the OC-EKF estimator both in simulation and experimentally.
\end{abstract}

\section{INTRODUCTION AND RELATED WORK}

In many robotic applications (e.g., search and rescue, environmental monitoring, planetary exploration, etc.), it is necessary to precisely know the robot's 3D position and orientation (pose). Over short periods of time, a robot can track its pose by fusing rotational velocity and linear acceleration measurements provided by an IMU. However, the integration of the bias and noise contaminating the IMU signals, makes the resulting estimates unreliable. For this reason, most inertial navigation systems (INS) rely on GPS measurements for bounding the estimation errors. For robots operating in GPS-denied areas, alternative sensors, such as cameras, have been employed for aiding INS, in what is known as vision-aided (V)-INS. Specifically, by processing observations of point features detected in images, a camera can provide periodic corrections to the inertial estimates (e.g., [1]). Although the small size, low weight and power requirements of cameras make them ideal for mobile robots, their inability to provide the depth to the scene poses certain challenges. In particular, a feature needs to be reliably tracked across multiple images and the baseline between them must be accurately estimated in order to determine the depth to it. Moreover, imprecise depth estimation can cause large linearization errors which can reduce the estimation accuracy and even lead to divergence.

An alternative to V-INS would be to fuse IMU measurements with observations from an RGBD camera, such as the Microsoft kinect [2], which directly measures the depth to the scene. To do so, however, one needs to precisely

The authors are with the Department of Computer Science \& Engineering, University of Minnesota, Minneapolis, MN 55455, USA \{chaguo|stergios\}@Cs. umn . edu

This work was supported by the University of Minnesota through the Digital Technology Center (DTC) and AFOSR MURI FA9550-10-1-0567. determine the 6 degrees of freedom (dof) transformation between the two sensors. Although numerous algorithms have been developed for IMU-noise characterization (e.g., [3]) and for intrinsically calibrating cameras (e.g., [4]) and RGBD sensors (e.g., [5]), to the best of our knowledge, there exists no algorithm for IMU-RGBD extrinsic calibration. Instead, one could potentially use methods designed for IMU-camera calibration (e.g., [6], [7], [8], [9], [10]). However, these would result in sub-optimal calibration accuracy since they would neglect the depth information available in the RGBD data. Furthermore, to date the conditions under which the IMU-RGBD calibration parameters are observable are not known. Previous efforts to study the observability properties of the IMU-camera calibration system have either relied on known calibration targets [8], or employed an inferred measurement model (i.e., assuming the camera observes its pose in the global frame, up to scale), which requires a nonminimal set of visual measurements [9].

In order to address these limitations, this paper makes the following key contributions:

- We study the observability of the nonlinear system describing the IMU-RGBD camera calibration and for the first time prove that although the system is unobservable, the unknown 6-dof transformation lies within its observable subspace and can be determined even when only one (previously unknown) point feature is detected. In this analysis, we do not make any assumptions about the prior location of the feature or the pose of the camera and do not rely on inferred camera measurements as is the case in [8], [9]. This is achieved by employing a new methodology for studying the observability of nonlinear systems [11] which employs appropriate change of variables for isolating the unobservable modes of the system.

- We analytically determine the vectors spanning the unobservable directions of the IMU-RGBD calibration system and use them in order to improve the consistency of the EKF designed for estimating the unknown calibration parameters.

- We validate the performance of the developed estimator, both in simulation and experimentally, when performing simultaneous localization, mapping, and IMU-RGBD calibration.

The rest of the paper is structured as follows. In Section IIA, we briefly describe the method of Hermann and Krener [12] for studying the observability of nonlinear systems and discuss the challenges for proving that a system is unobserv- 
able when following this method. In Section II-B, we present the approach introduced in [11] that employs change of variables to appropriately factorize the observability matrix and determine its unobservable directions. In Section III, we apply this method to the specific problem of IMURGBD calibration and show that although the calibration parameters are observable, the global position and rotation about the gravity vector are not. In Section IV, we present the observability-constrained (OC) EKF algorithm we developed for determining the unknown transformation and briefly discuss how its consistency [13] is improved using the results of Section III. In Section V, we assess the performance of the OC-EKF algorithm both in simulation and experimentally and verify its consistency. Finally, Section VI presents the conclusions of this work and provides possible directions of future research.

\section{NONLINEAR SySTEM OBSERVABILITY ANALYSIS}

In this section, we provide a brief overview of the method in [12] for studying the observability of nonlinear systems and then introduce a new methodology for determining its unobservable directions.

\section{A. Observability Analysis with Lie Derivatives}

Consider a nonlinear, continuous-time system:

$$
\left\{\begin{array}{l}
\dot{\mathbf{x}}=\mathbf{f}_{0}(\mathbf{x})+\sum_{i=1}^{l} \mathbf{f}_{i}(\mathbf{x}) u_{i} \\
\mathbf{y}=\mathbf{h}(\mathbf{x})
\end{array}\right.
$$

where $\mathbf{u}=\left[\begin{array}{lll}u_{1} & \ldots & u_{l}\end{array}\right]^{T}$ is its control input, $\mathbf{x}=$ $\left[\begin{array}{lll}x_{1} & \ldots & x_{m}\end{array}\right]^{T}$ is the system's state vector, $\mathbf{y}$ is the system output, and $\mathbf{f}_{i}, i=0, \ldots, l$ are the process functions. The zeroth-order Lie derivative of a measurement function $\mathbf{h}$ is defined as the function itself [12]:

$$
\mathfrak{L}^{0} \mathbf{h}=\mathbf{h}(\mathbf{x})
$$

while the span of the $i$ th order Lie derivative is defined as:

$$
\nabla \mathfrak{L}^{i} \mathbf{h}=\left[\begin{array}{llll}
\frac{\partial \mathfrak{L}^{i} \mathbf{h}}{\partial x_{1}} & \frac{\partial \mathfrak{L}^{i} \mathbf{h}}{\partial x_{2}} & \ldots & \frac{\partial \mathfrak{L}^{i} \mathbf{h}}{\partial x_{m}}
\end{array}\right]
$$

For any $i$ th order Lie derivative, $\mathfrak{L}^{i} \mathbf{h}$, the $i+1$ th order Lie derivative $\mathfrak{L}_{\mathbf{f}_{j}}^{i+1} \mathbf{h}$ with respect to a process function $\mathbf{f}_{j}$ can be computed as:

$$
\mathfrak{L}_{\mathbf{f}_{j}}^{i+1} \mathbf{h}=\nabla \mathfrak{L}^{i} \mathbf{h} \cdot \mathbf{f}_{j}
$$

The observability matrix $\mathcal{O}$ of system (1) is defined as a matrix with block rows the span of the Lie derivatives of (1), i.e.,

$$
\mathcal{O}=\left[\begin{array}{c}
\nabla \mathfrak{L}^{0} \mathbf{h} \\
\nabla \mathfrak{L}_{\mathbf{f}_{i}}^{1} \mathbf{h} \\
\nabla \mathfrak{L}_{\mathbf{f}_{i} \mathbf{f}_{j}}^{2} \mathbf{h} \\
\nabla \mathfrak{L}_{\mathbf{f}_{i} \mathbf{f}_{j} \mathbf{f}_{k}}^{3} \mathbf{h} \\
\vdots
\end{array}\right]
$$

where $i, j, k=0, \ldots, l$. Based on [12], to prove that a system is observable, it suffices to show that any submatrix of $\mathcal{O}$ comprising a subset of its rows is of full column rank. In contrast, to prove that a system is unobservable and find its unobservable directions, we need to: (i) show that the infinitely many block rows of $\mathcal{O}$ can be written as a linear combination of a subset of its block rows, which form a submatrix $\mathcal{O}^{\prime}$; and (ii) find the nullspace of $\mathcal{O}^{\prime}$ in order to determine the system's unobservable directions. Although accomplishing (ii) is fairly straightforward, achieving (i) is extremely challenging especially for high-dimensional systems, such as the one describing the IMU-RGBD camera calibration. If the system's unobservable directions are given, [14] provides a method to solve for the system's observable modes. However, this method does not overcome the difficulty of finding a nonlinear system's unobservable directions.

To address this issue, in the following section, we present a new methodology that relies on change of variables for proving that a system is unobservable and finding its unobservable directions. In addition, our proposed method directly determines the observable modes with which we can construct an observable system.

\section{B. Observability Analysis with Basis Functions}

We start by proving the following:

Theorem 1: Assume that there exists a nonlinear transformation $\boldsymbol{\beta}(\mathbf{x})=\left[\begin{array}{lll}\boldsymbol{\beta}_{1}(\mathbf{x})^{T} & \ldots & \boldsymbol{\beta}_{t}(\mathbf{x})^{T}\end{array}\right]^{T}$ (i.e., a set of basis functions) of the variable $\mathbf{x}$ in $(1)$, such that:

(A1) $\mathbf{h}(\mathbf{x})=\mathbf{h}^{\prime}(\boldsymbol{\beta})$ is a function of $\boldsymbol{\beta}$.

(A2) $\frac{\partial \boldsymbol{\beta}}{\partial \mathbf{x}} \cdot \mathbf{f}_{i}, i=0, \ldots, l$, are functions of $\boldsymbol{\beta}$;

(A3) $\boldsymbol{\beta}$ is a function of the variables of a set $\mathbf{S}$ comprising system (1) Lie derivatives from order zero up to order $p$, with $p<\infty$.

Then:

(i) The observability matrix of (1) can be factorized as: $\mathcal{O}=\Xi \cdot \mathbf{B}$, where $\mathbf{B} \triangleq \frac{\partial \boldsymbol{\beta}}{\partial \mathbf{x}}$ and $\Xi$ is the observability matrix of the following system (6).

$$
\left\{\begin{array}{l}
\dot{\boldsymbol{\beta}}=\mathbf{g}_{0}(\boldsymbol{\beta})+\sum_{i=1}^{l} \mathbf{g}_{i}(\boldsymbol{\beta}) u_{i} \\
\mathbf{y}=\mathbf{h}^{\prime}(\boldsymbol{\beta})
\end{array}\right.
$$

where $\mathbf{g}_{i}(\boldsymbol{\beta}) \triangleq \frac{\partial \boldsymbol{\beta}}{\partial \mathbf{x}} \mathbf{f}_{i}(\mathbf{x}), i=0, \ldots, l$.

(ii) System (6) is observable.

(iii) $\operatorname{null}(\mathcal{O})=\operatorname{null}(\mathbf{B})$.

Proof: (i) Based on the chain rule, the span of any Lie derivative $\nabla \mathfrak{L}^{i} \mathbf{h}$ can be written as:

$$
\nabla \mathfrak{L}^{i} \mathbf{h}=\frac{\partial \mathfrak{L}^{i} \mathbf{h}}{\partial \mathbf{x}}=\frac{\partial \mathfrak{L}^{i} \mathbf{h}}{\partial \boldsymbol{\beta}} \frac{\partial \boldsymbol{\beta}}{\partial \mathbf{x}}
$$

Thus the observability matrix $\mathcal{O}$ of (1) can be factorized as:

$$
\mathcal{O}=\left[\begin{array}{c}
\nabla \mathfrak{L}^{0} \mathbf{h} \\
\nabla \mathfrak{L}_{\mathbf{f}_{i}}^{1} \mathbf{h} \\
\nabla \mathfrak{L}_{\mathbf{f}_{i} \mathbf{f}_{j}}^{2} \mathbf{h} \\
\nabla \mathfrak{L}_{\mathbf{f}_{i} \mathbf{f}_{j} \mathbf{f}_{k}}^{3} \mathbf{h} \\
\vdots
\end{array}\right]=\left[\begin{array}{c}
\frac{\partial \mathfrak{L}^{0} \mathbf{h}}{\partial \boldsymbol{\beta}} \\
\frac{\partial \mathfrak{L}_{\mathbf{f}_{i}}^{1} \mathbf{h}}{\partial \boldsymbol{\beta}} \\
\frac{\partial \mathfrak{L}_{\mathbf{f}_{i} \mathbf{f}_{j}}^{2} \mathbf{h}}{\partial \boldsymbol{\beta}} \\
\frac{\partial \mathfrak{L}_{\mathbf{f}_{i} \mathbf{f}_{j} \mathbf{f}_{k}}^{3} \mathbf{h}}{\partial \boldsymbol{\beta}} \\
\vdots
\end{array}\right] \frac{\partial \boldsymbol{\beta}}{\partial \mathbf{x}}=\Xi \cdot \mathbf{B}
$$

Next we prove that $\Xi$ is the observability matrix of the system (6) by induction.

To distinguish the Lie derivatives of systems (1) and (6), let $\mathfrak{I}$ denote the Lie derivatives of system (6). Then, the span 
of its zeroth-order Lie derivative is:

$$
\nabla \mathfrak{I}^{0} \mathbf{h}^{\prime}=\nabla \mathfrak{I}^{0} \mathbf{h}=\frac{\partial \mathbf{h}}{\partial \boldsymbol{\beta}}=\frac{\partial \mathfrak{L}^{0} \mathbf{h}}{\partial \boldsymbol{\beta}}
$$

which corresponds to the first block row of $\Xi$.

Assume that the span of the $i$ th order Lie derivative of (6) along any direction can be written as $\nabla \mathfrak{I}^{i} \mathbf{h}^{\prime}=\frac{\partial \mathfrak{L}^{i} \mathbf{h}}{\partial \boldsymbol{\beta}}$, which corresponds to a block row of $\Xi$.

Then, the span of the $i+1$ th order Lie derivative $\nabla \mathfrak{I}_{\mathbf{g}_{j}}^{i+1} \mathbf{h}^{\prime}$ with respect to the process function $\mathbf{g}_{j}$ can be computed as:

$$
\begin{aligned}
\nabla \mathfrak{I}_{\mathbf{g}_{j}}^{i+1} \mathbf{h}^{\prime} & =\frac{\partial \mathfrak{I}_{\mathbf{g}_{j}}^{i+1} \mathbf{h}^{\prime}}{\partial \boldsymbol{\beta}}=\frac{\partial\left(\nabla \mathfrak{I}^{i} \mathbf{h}^{\prime} \cdot \mathbf{g}_{j}\right)}{\partial \boldsymbol{\beta}}=\frac{\partial\left(\frac{\partial \mathfrak{L}^{i} \mathbf{h}}{\partial \boldsymbol{\beta}} \cdot \frac{\partial \boldsymbol{\beta}}{\partial \mathbf{x}} \mathbf{f}_{j}(\mathbf{x})\right)}{\partial \boldsymbol{\beta}} \\
& =\frac{\partial\left(\frac{\partial \mathfrak{L}^{i} \mathbf{h}}{\partial \mathbf{x}} \cdot \mathbf{f}_{j}(\mathbf{x})\right)}{\partial \boldsymbol{\beta}}=\frac{\partial \mathfrak{L}_{\mathbf{f}_{j}}^{i+1} \mathbf{h}}{\partial \boldsymbol{\beta}}
\end{aligned}
$$

which is also the corresponding block row of $\Xi$. Therefore, we conclude that $\Xi$ is a matrix whose rows are the span of all the Lie derivatives of system (6), thus $\Xi$ is the observability matrix of system (6).

(ii) In (A3), $\boldsymbol{\beta}$ is defined as a function of variables in a set $\mathbf{S}$ comprising a subset of the Lie derivatives of system (1). Hence if we can prove that the Lie derivatives in set $\mathbf{S}$ are all observable, the state $\boldsymbol{\beta}$ of system (6) is also observable. In the following, we will show that the Lie derivatives included in $\mathbf{S}$, defined as $\mathbf{S} \triangleq$ $\left\{\mathfrak{L}^{0} \mathbf{h}, \quad \mathfrak{L}_{\mathbf{f}_{i}}^{1} \mathbf{h}, \quad \mathfrak{L}_{\mathbf{f}_{i} \mathbf{f}_{j}}^{2} \mathbf{h}, \quad \cdots, \quad \mathfrak{L}^{p} \mathbf{h}\right\}, \quad i, j=0, \ldots, l$, are all observable.

We start by multiplying both sides of the dynamic equation of system (1) with the span of the $j$ th-order Lie derivatives $\nabla \mathfrak{L}^{j} \mathbf{h}$ to obtain

$$
\nabla \mathfrak{L}^{j} \mathbf{h} \cdot \dot{\mathbf{x}}=\nabla \mathfrak{L}^{j} \mathbf{h} \cdot \mathbf{f}_{0}(\mathbf{x})+\sum_{i=1}^{l} \nabla \mathfrak{L}^{j} \mathbf{h} \cdot \mathbf{f}_{i}(\mathbf{x}) u_{i}
$$

Employing $\mathfrak{L}_{\mathbf{f}_{i}}^{j+1} \mathbf{h}=\nabla \mathfrak{L}^{j} \mathbf{h} \cdot \mathbf{f}_{i}$ (from the definition of Lie derivatives) and $\mathfrak{L}^{j} \mathbf{h}=\nabla \mathfrak{L}^{j} \mathbf{h} \cdot \dot{\mathbf{x}}$ (from the chain rule), we obtain:

$$
\mathfrak{L}^{j} \mathbf{h}=\mathfrak{L}_{\mathbf{f}_{0}}^{j+1} \mathbf{h}+\sum_{i=1}^{l} \mathfrak{L}_{f_{i}}^{j+1} \mathbf{h} \cdot u_{i}
$$

with which, we can construct a system in terms of all the Lie derivatives in $\mathbf{S}$ as:

$$
\begin{aligned}
& {\left[\begin{array}{c}
\mathfrak{L}^{0} \mathbf{h} \\
\mathfrak{L}_{\mathbf{f}_{i}}^{1} \mathbf{h} \\
\mathfrak{L}_{\mathbf{f}_{i} \mathbf{f}_{j}}^{2} \mathbf{h} \\
\vdots \\
\mathfrak{L}^{p-1} \mathbf{h}
\end{array}\right]=\left[\begin{array}{c}
\mathfrak{L}_{\mathbf{f}_{0}}^{1} \mathbf{h} \\
\mathfrak{L}_{\mathbf{f}_{i} \mathbf{f}_{0}}^{2} \mathbf{h} \\
\mathfrak{L}_{\mathbf{f}_{i} \mathbf{f}_{j} \mathbf{f}_{0}}^{3} \mathbf{h} \\
\vdots \\
\mathfrak{L}_{\mathbf{f}_{0}}^{p} \mathbf{h}
\end{array}\right]+\left[\begin{array}{c}
\sum_{t=1}^{l} \mathfrak{L}_{\mathbf{f}_{t}}^{1} \mathbf{h} \cdot u_{t} \\
\sum_{t=1}^{l} \mathfrak{L}_{\mathbf{f}_{i} \mathbf{f}_{t}}^{2} \mathbf{h} \cdot u_{t} \\
\sum_{t=1}^{l} \mathfrak{L}_{\mathbf{f}_{i} \mathbf{f}_{j} \mathbf{f}_{t}}^{3} \mathbf{h} \cdot u_{t} \\
\vdots \\
\sum_{t=1}^{l} \mathfrak{L}_{\mathbf{f}_{t}}^{p} \mathbf{h} \cdot u_{t}
\end{array}\right]} \\
& \mathbf{y}=\mathfrak{L}^{0} \mathbf{h}
\end{aligned}
$$

By directly computing its Lie derivatives, it is straightforward to show that the observability matrix of system (13) contains an identity matrix at the top, thus it is of full column rank. Therefore, all the Lie derivatives in $\mathbf{S}$ are observable. Due to the fact that the basis function $\boldsymbol{\beta}$ is a function of the Lie derivatives in $\mathbf{S}$ (see (A3)), system (6) is also observable.

(iii) From $\mathcal{O}=\Xi \cdot \mathbf{B}$, we have the relation $\operatorname{null}(\mathcal{O})=$
$\operatorname{null}(\mathbf{B})+\operatorname{null}(\Xi) \cap$ range $(\mathbf{B})$. Since we have proved system (6) is observable, its observability matrix $\Xi$ is of full column rank. Therefore, we have $\operatorname{null}(\mathcal{O})=\operatorname{null}(\mathbf{B})$.

Based on Theorem 1, the unobservable directions can be determined with much less effort. To find a system's unobservable directions, we first need to define the basis functions that satisfy conditions $(A 1)$ and $(A 3)$, and verify that condition $(A 2)$ is satisfied, or equivalently that the basis function set is complete. Once all the conditions are fulfilled, the unobservable directions of (1) correspond to the nullspace of matrix $\mathbf{B}$, which has finite dimensions, and thus it is easy to analyze.

In the following section, we will leverage Theorem 1 to prove that the IMU-RGBD camera calibration system is unobservable and find its unobservable directions. To do this, in Section III-A we first present the formulation of the IMURGBD camera calibration nonlinear system. In Section III$B$, we find the set of basis functions that satisfies the three conditions of Theorem 1. In Section III-C, we construct an observable system in terms of the basis functions. In Section III-D, we determine the unobservable directions of the IMU-RGBD camera calibration system by finding the nullspace of matrix $\mathbf{B}$.

\section{OBSERVABILITY ANALYSIS OF THE IMU-RGBD CAMERA 3D POSE ESTIMATION AND EXTRINSIC Calibration System}

In this section, we present the observability analysis for the IMU-RGBD camera 3D pose estimation and extrinsic calibration problem using basis functions. Since the Lie derivatives for all the landmarks have identical form, for simplicity we keep only one landmark in the state vector. The extension to multiple landmarks is described in [15].

\section{A. System Model}

In the IMU-RGBD camera 3D pose estimation and extrinsic calibration problem, the state vector we estimate is:

$$
\mathbf{x}=\left[\begin{array}{lllllllll}
{ }^{I} \mathbf{s}_{G}^{T} & { }^{G} \mathbf{v}_{I}^{T} & { }^{G} \mathbf{p}_{I}^{T} & { }^{G} \mathbf{p}_{f}^{T} & \mathbf{b}_{a}^{T} & \mathbf{b}_{g}^{T} & { }^{C} \mathbf{S}_{I}^{T} & { }^{I} \mathbf{p}_{C}^{T}
\end{array}\right]^{T}
$$

where ${ }^{I} \mathbf{S}_{G}$ is the Cayley-Gibbs-Rodriguez parameterization [16] representing the orientation of the global frame $\{G\}$ in the IMU's frame of reference $\{I\},{ }^{G} \mathbf{v}_{I}$ and ${ }^{G} \mathbf{p}_{I}$ represent the velocity and position of $\{I\}$ in $\{G\}, \mathbf{b}_{a}$ and $\mathbf{b}_{g}$ are the biases in the gyroscope and accelerometer measurements, ${ }^{C} \mathbf{S}_{I}$ represents the orientation of $\{I\}$ in the RGBD camera's frame of reference $\{C\},{ }^{I} \mathbf{p}_{C}$ is the position of $\{C\}$ in $\{I\}$, and ${ }^{G} \mathbf{p}_{f}$ represents the estimated landmark in $\{G\}$.

1) System propagation model: The system model describing the time evolution of the states is:

$$
\begin{aligned}
{ }^{I} \dot{\mathbf{s}}_{G}(t) & =\frac{1}{2} \mathbf{D}\left({ }^{I} \boldsymbol{\omega}(t)-\mathbf{b}_{g}(t)\right) \\
{ }^{G} \dot{\mathbf{v}}_{I}(t) & ={ }^{G} \mathbf{a}(t)={ }^{G} \mathbf{g}+\mathbf{C}\left({ }^{I} \mathbf{s}_{G}(t)\right)^{T}\left({ }^{I} \mathbf{a}(t)-\mathbf{b}_{a}(t)\right) \\
{ }^{G} \dot{\mathbf{p}}_{I}(t) & ={ }^{G} \mathbf{v}_{I}(t) \quad{ }^{G} \dot{\mathbf{p}}_{f}(t)=\mathbf{0}_{3 \times 1} \\
\dot{\mathbf{b}}_{a}(t) & =\mathbf{0}_{3 \times 1} \quad \dot{\mathbf{b}}_{g}(t)=\mathbf{0}_{3 \times 1} \\
{ }^{C} \dot{\mathbf{s}}_{I}(t) & =\mathbf{0}_{3 \times 1} \quad{ }^{I} \dot{\mathbf{p}}_{C}(t)=\mathbf{0}_{3 \times 1}
\end{aligned}
$$


where $\mathbf{C}(\mathbf{s})$ represents the corresponding rotation matrix of $\mathbf{s},{ }^{I} \boldsymbol{\omega}(t)=\left[\begin{array}{lll}\omega_{1} & \omega_{2} & \omega_{3}\end{array}\right]^{T}$ and ${ }^{I} \mathbf{a}(t)=\left[\begin{array}{lll}a_{1} & a_{2} & a_{3}\end{array}\right]^{T}$ are the IMU rotational velocity and linear acceleration expressed in $\{I\}, \mathbf{b}_{g}(t)$ and $\mathbf{b}_{a}(t)$ are the gyroscope and accelerometer biases, $\mathbf{D} \triangleq 2 \frac{\partial \mathbf{s}}{\partial \boldsymbol{\theta}}=\mathbf{I}+\lfloor\mathbf{s} \times\rfloor+\mathbf{s} \mathbf{s}^{T}$, where $\boldsymbol{\theta}=\alpha \mathbf{k}$ represents a rotation by an angle $\alpha$ around the unit vector $\mathbf{k}$.

2) System measurement model: The RGBD camera can directly measure the $3 \mathrm{D}$ position of the landmark ${ }^{C} \mathbf{p}_{f}$ in its own frame of reference as:

$$
\mathbf{z}={ }^{C} \mathbf{p}_{f}=\mathbf{C}\left({ }^{C} \mathbf{s}_{I}\right) \mathbf{C}\left({ }^{I} \mathbf{s}_{G}\right)\left({ }^{G} \mathbf{p}_{f}-{ }^{G} \mathbf{p}_{I}\right)-\mathbf{C}\left({ }^{C} \mathbf{s}_{I}\right){ }^{I} \mathbf{p}_{C}
$$

\section{B. Determining the System's Basis Functions}

In this section, we find basis functions based on the conditions $(A 1)$ and $(A 3)$ of Theorem 1, until condition (A2) is satisfied for all the defined basis functions.

For simplicity, we retain only a few of the subscripts and superscripts in the state elements and write the system state vector as:

$$
\mathbf{x}=\left[\begin{array}{llllllll}
\mathbf{s}^{T} & \mathbf{v}^{T} & \mathbf{p}^{T} & \mathbf{p}_{f}^{T} & \mathbf{b}_{a}^{T} & \mathbf{b}_{g}^{T} & \mathbf{s}_{C}^{T} & \mathbf{p}_{C}^{T}
\end{array}\right]^{T}
$$

Then the IMU-RGBD camera 3D pose estimation and extrinsic calibration system can be rewritten as:

$$
\begin{aligned}
& {\left[\begin{array}{c}
\dot{\mathbf{s}} \\
\dot{\mathbf{v}} \\
\dot{\mathbf{p}} \\
\dot{\mathbf{p}}_{f} \\
\dot{\mathbf{b}}_{a} \\
\dot{\mathbf{b}}_{g} \\
\dot{\mathbf{S}}_{C} \\
\dot{\mathbf{p}}_{C}
\end{array}\right]=\underbrace{\left[\begin{array}{c}
-\frac{1}{2} \mathbf{D b}_{g} \\
\mathbf{g}-\mathbf{C}^{T} \mathbf{b}_{a} \\
\mathbf{v} \\
\mathbf{0} \\
\mathbf{0} \\
\mathbf{0} \\
\mathbf{0} \\
\mathbf{0}
\end{array}\right]}_{f_{0}}+\underbrace{\left[\begin{array}{c}
\frac{1}{2} \mathbf{D} \\
\mathbf{0} \\
\mathbf{0} \\
\mathbf{0} \\
\mathbf{0} \\
\mathbf{0} \\
\mathbf{0} \\
\mathbf{0}
\end{array}\right]}_{\mathbf{f}_{1}} \boldsymbol{\omega}+\underbrace{\left[\begin{array}{c}
\mathbf{0} \\
\mathbf{C}^{T} \\
\mathbf{0} \\
\mathbf{0} \\
\mathbf{0} \\
\mathbf{0} \\
\mathbf{0} \\
\mathbf{0}
\end{array}\right]}_{\mathbf{f}_{2}} \mathbf{a}} \\
& \mathbf{z}=\mathbf{C}_{C} \mathbf{C}\left(\mathbf{p}_{f}-\mathbf{p}\right)-\mathbf{C}_{C} \mathbf{p}_{C}
\end{aligned}
$$

where $\mathbf{C} \triangleq \mathbf{C}(\mathbf{s}), \mathbf{C}_{C} \triangleq \mathbf{C}\left(\mathbf{s}_{C}\right)$. Note that $\mathbf{f}_{0}$ is a $24 \times 1$ vector, while $\mathbf{f}_{1}$ and $\mathbf{f}_{2}$ are both $24 \times 3$ matrices which is a compact way for representing three process functions:

$$
\begin{aligned}
\mathbf{f}_{1} \boldsymbol{\omega} & =f_{11} \omega_{1}+f_{12} \omega_{2}+f_{13} \omega_{3} \\
\mathbf{f}_{2} \mathbf{a} & =f_{21} a_{1}+f_{22} a_{2}+f_{23} a_{3}
\end{aligned}
$$

To define the basis functions for this system, we follow the conditions of Theorem 1: (i) Select basis functions so that the measurement function $\mathbf{z}$ can be expressed as a function of $\boldsymbol{\beta}$; (ii) Select the remaining basis functions as functions of the system's Lie derivatives, until condition (A2), (i.e., $\frac{\partial \boldsymbol{\beta}}{\partial \mathbf{x}} \cdot \mathbf{f}_{i}$ is a function of $\boldsymbol{\beta}$ for any $i$ ), is satisfied by all the basis functions. 1

For this problem, we define the first basis function directly as the measurement function:

$$
\boldsymbol{\beta}_{1} \triangleq \mathbf{z}=\mathbf{C}_{C} \mathbf{C}\left(\mathbf{p}_{f}-\mathbf{p}\right)-\mathbf{C}_{C} \mathbf{p}_{C}
$$

where $\boldsymbol{\beta}_{1}$ is a $3 \times 1$ vector representing in a compact form 3 basis functions. To check if the condition (A2) of Theorem 1

\footnotetext{
${ }^{1}$ Note that although the definition of the basis functions is not unique, any basis functions that satisfy the conditions of Theorem 1 span the same space.
}

is fulfilled, we compute the span of $\boldsymbol{\beta}_{1}$ with respect to $\mathbf{x}$

$$
\begin{aligned}
\frac{\partial \boldsymbol{\beta}_{1}}{\partial \mathbf{x}} & =\left[\begin{array}{llllllll}
\frac{\partial \boldsymbol{\beta}_{1}}{\partial \boldsymbol{\theta}} \frac{\partial \boldsymbol{\theta}}{\partial \mathbf{s}} & \frac{\partial \boldsymbol{\beta}_{1}}{\partial \mathbf{v}} & \frac{\partial \boldsymbol{\beta}_{1}}{\partial \mathbf{p}} & \frac{\partial \boldsymbol{\beta}_{1}}{\partial \mathbf{p}_{f}} & \frac{\partial \boldsymbol{\beta}_{1}}{\partial \mathbf{b}_{a}} & \frac{\partial \boldsymbol{\beta}_{1}}{\partial \mathbf{b}_{g}} & \frac{\partial \boldsymbol{\beta}_{1}}{\partial \mathbf{s}_{C}} & \frac{\partial \boldsymbol{\beta}_{1}}{\partial \mathbf{p}_{C}}
\end{array}\right] \\
& =\left[\begin{array}{llllllll}
\mathbf{C}_{C}\left\lfloor\mathbf{C}\left(\mathbf{p}_{f}-\mathbf{p}\right)\right\rfloor \frac{\partial \boldsymbol{\theta}}{\partial \mathbf{s}} & \mathbf{0} & -\mathbf{C}_{C} \mathbf{C} & \mathbf{C}_{C} \mathbf{C} & \mathbf{0} & \mathbf{0} & \left\lfloor\beta_{1}\right\rfloor \frac{\partial \boldsymbol{\theta}_{C}}{\partial \mathbf{s}_{C}} & -\mathbf{C}_{C}
\end{array}\right]
\end{aligned}
$$

and project it onto all the process functions:

$$
\begin{aligned}
\frac{\partial \boldsymbol{\beta}_{1}}{\partial \mathbf{x}} \cdot f_{0} & =-\mathbf{C}_{C}\left\lfloor\mathbf{C}\left(\mathbf{p}_{f}-\mathbf{p}\right)\right\rfloor \mathbf{b}_{g}-\mathbf{C}_{C} \mathbf{C v} \\
\frac{\partial \boldsymbol{\beta}_{1}}{\partial \mathbf{x}} \cdot f_{1 i} & =\mathbf{C}_{C}\left\lfloor\mathbf{C}\left(\mathbf{p}_{f}-\mathbf{p}\right)\right\rfloor \mathbf{e}_{i} \\
& =\left\lfloor\mathbf{C}_{C} \mathbf{C}\left(\mathbf{p}_{f}-\mathbf{p}\right)\right\rfloor \mathbf{C}_{C} \mathbf{e}_{i} \\
\frac{\partial \boldsymbol{\beta}_{1}}{\partial \mathbf{x}} \cdot f_{2 i} & =\mathbf{0}
\end{aligned}
$$

where $i=1,2,3, \mathbf{e}_{1}=\left[\begin{array}{lll}1 & 0 & 0\end{array}\right]^{T}, \mathbf{e}_{2}=\left[\begin{array}{lll}0 & 1 & 0\end{array}\right]^{T}$, $\mathbf{e}_{3}=\left[\begin{array}{lll}0 & 0 & 1\end{array}\right]^{T}, \frac{\partial \boldsymbol{\theta}}{\partial \mathbf{s}} \frac{1}{2} \mathbf{D}=\frac{\partial \boldsymbol{\theta}}{\partial \mathbf{s}} \frac{\partial \mathbf{s}}{\partial \boldsymbol{\theta}}=\mathbf{I}_{3}$.

Obviously, $\frac{\partial \boldsymbol{\beta}_{1}}{\partial \mathbf{x}} \cdot f_{0}$ and $\frac{\partial \boldsymbol{\beta}_{1}}{\partial \mathbf{x}} \cdot f_{1 i}$ are not functions of the defined basis function $\boldsymbol{\beta}_{1}$. To proceed, we will employ condition (A3) of Theorem 1 to define additional basis functions as nonlinear combinations of the system's Lie derivatives.

Since the basis function $\boldsymbol{\beta}_{1}$ equals to the zeroth-order Lie derivative, then by definition, (19)-(21) are the first-order Lie derivatives:

$$
\frac{\partial \boldsymbol{\beta}_{1}}{\partial \mathbf{x}} \cdot f_{0}=\mathfrak{L}_{\mathbf{f}_{0}}^{1} \mathbf{h} \quad \frac{\partial \boldsymbol{\beta}_{1}}{\partial \mathbf{x}} \cdot f_{1 i}=\mathfrak{L}_{\mathbf{f}_{1 i}}^{1} \mathbf{h} \quad \frac{\partial \boldsymbol{\beta}_{1}}{\partial \mathbf{x}} \cdot f_{2 i}=\mathfrak{L}_{\mathbf{f}_{2 i}}^{1} \mathbf{h}
$$

Hereafter, we will make use of these Lie derivatives, which are observable, to find more basis functions. First, by multiplying $\left(\mathbf{C}_{C} \mathbf{e}_{i}\right)^{T}$ to both sides of (20), we have:

$$
\begin{aligned}
& \left(\mathbf{C}_{C} \mathbf{e}_{i}\right)^{T} \mathfrak{L}_{\mathbf{f}_{1 i}^{1}}^{1} \mathbf{h} \\
= & \left(\mathbf{C}_{C} \mathbf{e}_{i}\right)^{T}\left\lfloor\mathbf{C}_{C} \mathbf{C}\left(\mathbf{p}_{f}-\mathbf{p}\right)\right\rfloor \mathbf{C}_{C} \mathbf{e}_{i}=0 \quad i=1,2,3
\end{aligned}
$$

Since $\mathbf{C}_{C}=\mathbf{C}\left(\mathbf{s}_{C}\right)$, (22) is a quadratic equation in the three elements of $\mathbf{s}_{C}$ for which, up to eight solutions can be found for $\mathbf{s}_{C}$ in terms of the Lie derivatives $\mathfrak{L}_{\mathbf{f}_{1 i}}^{1} \mathbf{h}$ [17]. Therefore, we select the Cayley-Gibbs-Rodriguez parameterization of the rotation between IMU and RGBD camera as a new basis function.

$$
\boldsymbol{\beta}_{2} \triangleq \mathbf{s}_{C}
$$

Furthermore, we stack equation (20), for $i=1,2,3$, into a matrix form as:

$$
\left[\begin{array}{c}
\mathfrak{L}_{\mathbf{f}_{11}^{1}}^{1} \mathbf{h} \\
\mathfrak{L}_{\mathbf{f}_{12} \mathbf{h}}^{1} \\
\mathfrak{L}_{\mathbf{f}_{13}}^{1} \mathbf{h}
\end{array}\right]=-\underbrace{\left[\begin{array}{l}
\left\lfloor\mathbf{C}_{C} \mathbf{e}_{1}\right\rfloor \\
\left\lfloor\mathbf{C}_{C} \mathbf{e}_{2}\right\rfloor \\
\left\lfloor\mathbf{C}_{C} \mathbf{e}_{3}\right\rfloor
\end{array}\right]}_{\mathbf{Y}} \mathbf{C}_{C} \mathbf{C}\left(\mathbf{p}_{f}-\mathbf{p}\right)
$$

Since $\mathbf{Y}$ is a $9 \times 3$ matrix of full column rank, $\mathbf{C}_{C} \mathbf{C}\left(\mathbf{p}_{f}-\right.$ p) can be determined in terms of the Lie derivatives $\mathfrak{L}_{\mathbf{f}_{1 i}}^{1} \mathbf{h}$ and $\boldsymbol{\beta}_{2}$. Therefore, we define $\mathbf{C}_{C} \mathbf{C}\left(\mathbf{p}_{f}-\mathbf{p}\right)$ as a new basis function:

$$
\boldsymbol{\beta}_{3} \triangleq \mathbf{C}_{C} \mathbf{C}\left(\mathbf{p}_{f}-\mathbf{p}\right)
$$

After defining $\boldsymbol{\beta}_{2}$ and $\boldsymbol{\beta}_{3}$, note (19) still contains terms not belonging to the defined basis function set, thus the condition (A2) is not satisfied. To solve this problem, we have to find new basis functions by employing higher-order 
Lie derivatives. By definition, $\mathfrak{L}_{\mathbf{f}_{0} \mathbf{f}_{1 i}}^{2} \mathbf{h}$ can be obtained as:

$\mathfrak{L}_{\mathbf{f}_{0} \mathbf{f}_{1 i}}^{2} \mathbf{h}=\mathbf{C}_{C}\left\lfloor\mathbf{b}_{g}\right\rfloor\left\lfloor\mathbf{C}\left(\mathbf{p}_{f}-\mathbf{p}\right)\right\rfloor \mathbf{e}_{i}-\mathbf{C}_{C}\lfloor\mathbf{C v}\rfloor \mathbf{e}_{i} \quad i=1,2,3$

stacking these equations in a matrix form, we have:

$$
\underbrace{\left[\begin{array}{ll}
\left.-\left\lfloor\mathbf{C}\left(\mathbf{p}_{f}-\mathbf{p}\right)\right\rfloor \mathbf{e}_{1}\right\rfloor & \left\lfloor\mathbf{e}_{1}\right\rfloor \\
\left.-\left\lfloor\mathbf{C}\left(\mathbf{p}_{f}-\mathbf{p}\right)\right\rfloor \mathbf{e}_{2}\right\rfloor & \left\lfloor\mathbf{e}_{2}\right\rfloor \\
\left.-\left\lfloor\mathbf{C}\left(\mathbf{p}_{f}-\mathbf{p}\right)\right\rfloor \mathbf{e}_{3}\right\rfloor & \left\lfloor\mathbf{e}_{3}\right\rfloor
\end{array}\right]}_{\mathbf{V}}\left[\begin{array}{c}
\mathbf{b}_{g} \\
\mathbf{C v}
\end{array}\right]=\left[\begin{array}{l}
\mathbf{C}_{C}^{T} \mathfrak{L}_{\mathbf{f}_{0} \mathbf{f}_{11}}^{2} \mathbf{h} \\
\mathbf{C}_{C}^{T} \mathfrak{L}_{\mathbf{f}_{0} \mathbf{f}_{12}}^{2} \mathbf{h} \\
\mathbf{C}_{C}^{T} \mathfrak{L}_{\mathbf{f}_{0}}^{2} \mathbf{\mathbf { f } _ { 1 3 }} \mathbf{h}
\end{array}\right]
$$

in which

$$
\mathbf{C}_{C}=\mathbf{C}\left(\boldsymbol{\beta}_{2}\right), \quad \mathbf{C}\left(\mathbf{p}_{f}-\mathbf{p}\right)=\mathbf{C}\left(\boldsymbol{\beta}_{2}\right)^{T} \boldsymbol{\beta}_{3}
$$

are both expressed in terms of the previously defined basis functions, and thus are also functions of the Lie derivatives. $\mathbf{V}$ is a $9 \times 6$ matrix and it is easy to see that $\mathbf{V}$ is of full column rank, in general, using Gaussian elimination. Therefore, $\mathbf{b}_{g}$ and $\mathbf{C v}$ can be both determined in terms of the Lie derivatives, hence we define them as new basis functions:

$$
\boldsymbol{\beta}_{4} \triangleq \mathbf{b}_{g}, \quad \boldsymbol{\beta}_{5} \triangleq \mathbf{C v}
$$

After finding $\boldsymbol{\beta}_{4}$ and $\boldsymbol{\beta}_{5}$, (19) can now be expressed in terms of the basis functions.

We proceed by projecting the span of $\boldsymbol{\beta}_{5}$ onto the process function $f_{0}$ :

$$
\frac{\partial \boldsymbol{\beta}_{5}}{\partial \mathbf{x}} \cdot f_{0}=-\lfloor\mathbf{C v}\rfloor \mathbf{b}_{g}+\mathbf{C g}-\mathbf{b}_{a}
$$

and note that $\mathbf{C g}$ and $\mathbf{b}_{a}$ are not functions of the defined basis functions, thus condition (A2) is not fulfilled. Therefore, our basis function set is not complete yet. Next we will show how to find the missing basis functions from other Lie derivatives. By definition, we obtain the third-order Lie derivative $\mathfrak{L}_{\mathbf{f}_{0} \mathbf{f}_{0} \mathbf{f}_{1 i}}^{3} \mathbf{h}$ as:

$$
\begin{aligned}
\mathfrak{L}_{\mathbf{f}_{0} \mathbf{f}_{0} \mathbf{f}_{1 i}}^{3} \mathbf{h}= & \mathbf{C}_{C}\left\lfloor\mathbf{b}_{g}\right\rfloor^{2}\left\lfloor\mathbf{C}\left(\mathbf{p}_{f}-\mathbf{p}\right)\right\rfloor \mathbf{e}_{i}+2 \mathbf{C}_{C}\left\lfloor\mathbf{b}_{g}\right\rfloor\left\lfloor\mathbf{e}_{i}\right\rfloor \mathbf{C v}+ \\
& \mathbf{C}_{C}\left\lfloor\mathbf{e}_{i}\right\rfloor \mathbf{C g}, \quad i=1,2,3
\end{aligned}
$$

Using equation (30), for $i=1,2, \mathbf{C g}$ can be written in terms of $\mathfrak{L}_{\mathbf{f}_{0} \mathbf{f}_{0} \mathbf{f}_{1 i}}^{3} \mathbf{h}, \mathbf{C}_{C}, \mathbf{b}_{g}, \mathbf{C}\left(\mathbf{p}_{f}-\mathbf{p}\right), \mathbf{C v}, \mathbf{C g}$, which are all functions of Lie derivatives. Therefore, we can define $\mathbf{C g}$ as a new basis function:

$$
\boldsymbol{\beta}_{6} \triangleq \mathbf{C g}
$$

Subsequently, we consider the second-order Lie derivative $\mathfrak{L}_{\mathbf{f}_{0} \mathbf{f}_{0}}^{2} \mathbf{h}$ :

$$
\begin{aligned}
\mathfrak{L}_{\mathbf{f}_{0} \mathbf{f}_{0}}^{2} \mathbf{h}= & -\mathbf{C}_{C}\left\lfloor\mathbf{b}_{g}\right\rfloor\left\lfloor\mathbf{C}\left(\mathbf{p}_{f}-\mathbf{p}\right)\right\rfloor \mathbf{b}_{g}-\mathbf{C}_{C} \mathbf{C g} \\
& +\mathbf{C}_{C} \mathbf{b}_{a}-2 \mathbf{C}_{C}\left\lfloor\mathbf{b}_{g}\right\rfloor \mathbf{C v}
\end{aligned}
$$

and note that $\mathbf{b}_{a}$ can be uniquely determined from $\mathfrak{L}_{\mathbf{f}_{0} \mathbf{f}_{0}}^{2} \mathbf{h}$, $\mathbf{C}_{C}, \mathbf{b}_{g}, \mathbf{C}\left(\mathbf{p}_{f}-\mathbf{p}\right), \mathbf{C v}, \mathbf{C g}$, which are all functions of Lie derivatives. Thus, it satisfies condition (A3) and we define it as a new basis function:

$$
\boldsymbol{\beta}_{7} \triangleq \mathbf{b}_{a}
$$

Up to this point, we have defined a set of basis functions, and it is easy to verify that all three conditions of Theorem 1 are satisfied. Therefore, we have found the basis functions spanning the observable space of the IMU-RGBD camera system.

\section{Observable System in Terms of Basis Functions}

Since all of the previously defined basis functions are functions of the Lie derivatives, the terms defined by any operation between them remain functions of the Lie derivatives. Therefore, we remove some redundant terms from the previously defined basis functions, and redefine a new basis function set as:

$$
\boldsymbol{\beta}^{\prime}=\left[\begin{array}{c}
\boldsymbol{\beta}_{1}^{\prime} \\
\boldsymbol{\beta}_{2}^{\prime} \\
\boldsymbol{\beta}_{3}^{\prime} \\
\boldsymbol{\beta}_{4}^{\prime} \\
\boldsymbol{\beta}_{5}^{\prime} \\
\boldsymbol{\beta}_{6}^{\prime} \\
\boldsymbol{\beta}_{7}^{\prime}
\end{array}\right] \triangleq\left[\begin{array}{c}
\mathbf{C}\left(\boldsymbol{\beta}_{2}\right)^{T} \boldsymbol{\beta}_{3} \\
\mathbf{C}\left(\boldsymbol{\beta}_{2}\right)^{T}\left(\boldsymbol{\beta}_{3}-\boldsymbol{\beta}_{1}\right) \\
\boldsymbol{\beta}_{2} \\
\boldsymbol{\beta}_{4} \\
\boldsymbol{\beta}_{5} \\
\boldsymbol{\beta}_{6} \\
\boldsymbol{\beta}_{7}
\end{array}\right]=\left[\begin{array}{c}
\mathbf{C}\left(\mathbf{p}_{f}-\mathbf{p}\right) \\
\mathbf{p}_{C} \\
\mathbf{s}_{C} \\
\mathbf{b}_{g} \\
\mathbf{C v} \\
\mathbf{C g} \\
\mathbf{b}_{a}
\end{array}\right]
$$

With this new defined basis function set, we leverage conclusion (i) of Theorem 1 to construct the observable system in terms of the basis functions as:

$$
\begin{aligned}
\dot{\boldsymbol{\beta}}^{\prime} & =\frac{\partial \boldsymbol{\beta}^{\prime}}{\partial \mathbf{x}} \dot{\mathbf{x}}=\frac{\partial \boldsymbol{\beta}^{\prime}}{\partial \mathbf{x}}\left(\mathbf{f}_{0}(\mathbf{x})+\sum_{i=1}^{l} \mathbf{f}_{i}(\mathbf{x}) u_{i}\right) \\
= & {\left[\begin{array}{c}
\left.-\left\lfloor\mathbf{C}\left(\mathbf{p}_{f}-\mathbf{p}\right)\right\rfloor \mathbf{b}_{g}-\mathbf{C v}\right] \\
\mathbf{0} \\
\mathbf{0} \\
\mathbf{0} \\
-\lfloor\mathbf{C v}\rfloor \mathbf{b}_{g}+\mathbf{C g}-\mathbf{b}_{a} \\
-\lfloor\mathbf{C g}\rfloor \mathbf{b}_{g} \\
\mathbf{0}
\end{array}\right]+\left[\begin{array}{c}
\left\lfloor\mathbf{C}\left(\mathbf{p}_{f}-\mathbf{p}\right)\right\rfloor \\
\mathbf{0} \\
\mathbf{0} \\
\mathbf{0} \\
\lfloor\mathbf{C v}\rfloor \\
{[\mathbf{C g}\rfloor} \\
\mathbf{0}
\end{array}\right] \boldsymbol{\omega}+\left[\begin{array}{l}
\mathbf{0} \\
\mathbf{0} \\
\mathbf{0} \\
\mathbf{I} \\
\mathbf{0} \\
\mathbf{0}
\end{array}\right] \mathbf{a} } \\
= & {\left[\begin{array}{c}
-\left\lfloor\boldsymbol{\beta}_{1}^{\prime}\right\rfloor \boldsymbol{\beta}_{4}^{\prime}-\boldsymbol{\beta}_{5}^{\prime} \\
\mathbf{0} \\
\mathbf{0} \\
\mathbf{0} \\
-\left\lfloor\boldsymbol{\beta}_{4}^{\prime}+\boldsymbol{\beta}_{6}^{\prime}-\boldsymbol{\beta}_{7}^{\prime}\right. \\
\mathbf{0}
\end{array}\right]+\left[\begin{array}{c}
\mathbf{0} \\
\mathbf{0} \\
\mathbf{0} \\
\mathbf{0} \\
\left\lfloor\boldsymbol{\beta}_{5}^{\prime}\right\rfloor \\
{\left[\boldsymbol{\beta}_{6}^{\prime}\right\rfloor} \\
\mathbf{0}
\end{array}\right] \boldsymbol{\omega}+\left[\begin{array}{l}
\mathbf{0} \\
\mathbf{0} \\
\mathbf{I} \\
\mathbf{0} \\
\mathbf{0}
\end{array}\right] \mathbf{a} }
\end{aligned}
$$

Note that even if more than the minimum number of required basis functions are defined, it does not affect our analysis except that the defined observable system in terms of the basis functions is not a minimal system. In the following, we will show how to find the unobservable directions of the IMURGBD camera 3D pose estimation and extrinsic calibration system by leveraging conclusion (iii) of Theorem 1 .

\section{Determining the System's Observability Matrix}

In this section, we determine the unobservable directions of system (16) by finding the nullspace of the basis functions' span $\mathbf{B}=\frac{\partial \boldsymbol{\beta}^{\prime}}{\partial \mathbf{x}}$.

Theorem 2: The IMU-RGBD camera 3D pose estimation and extrinsic calibration system (16) is unobservable, and its unobservable sub-space is spanned by 4 directions corresponding to the IMU-RGBD camera global position and its rotation around the gravity vector in the global frame.

Proof: In the previous section, we have proved that the basis function $\boldsymbol{\beta}^{\prime}$ satisfies all three conditions of Theorem 1 . Therefore, $\operatorname{null}(\mathcal{O})=\operatorname{null}(\mathbf{B})$, (i.e., the system's unobservable directions span the nullspace of matrix $\mathbf{B}$ ), and we can determine the observability properties of the IMU-RGBD 
camera system by analyzing the rank condition of matrix $\mathbf{B}$. Stacking the spans of the basis functions $\boldsymbol{\beta}^{\prime}$ with respect to the system state $\mathbf{x}$, the matrix $\mathbf{B}$ is defined as:

$$
\left[\begin{array}{cccccccc}
\left\lfloor\mathbf{C}\left(\mathbf{p}_{f}-\mathbf{p}\right)\right\rfloor \frac{\partial \boldsymbol{\theta}}{\partial \mathbf{s}} & \mathbf{0} & -\mathbf{C} & \mathbf{C} & \mathbf{0} & \mathbf{0} & \mathbf{0} & \mathbf{0} \\
\mathbf{0} & \mathbf{0} & \mathbf{0} & \mathbf{0} & \mathbf{0} & \mathbf{0} & \mathbf{0} & \mathbf{I} \\
\mathbf{0} & \mathbf{0} & \mathbf{0} & \mathbf{0} & \mathbf{0} & \mathbf{0} & \mathbf{I} & \mathbf{0} \\
\mathbf{0} & \mathbf{0} & \mathbf{0} & \mathbf{0} & \mathbf{0} & \mathbf{I} & \mathbf{0} & \mathbf{0} \\
\lfloor\mathbf{C V}\rfloor \frac{\partial \boldsymbol{\theta}}{\partial \mathrm{s}} & \mathbf{C} & \mathbf{0} & \mathbf{0} & \mathbf{0} & \mathbf{0} & \mathbf{0} & \mathbf{0} \\
\lfloor\mathbf{C g}\rfloor \frac{\partial \boldsymbol{\theta}}{\partial \mathbf{s}} & \mathbf{0} & \mathbf{0} & \mathbf{0} & \mathbf{0} & \mathbf{0} & \mathbf{0} & \mathbf{0} \\
\mathbf{0} & \mathbf{0} & \mathbf{0} & \mathbf{0} & \mathbf{I} & \mathbf{0} & \mathbf{0} & \mathbf{0}
\end{array}\right]
$$

Let $\mathbf{A}=\left[\begin{array}{llllllll}\mathbf{A}_{1}^{T} & \mathbf{A}_{2}^{T} & \mathbf{A}_{3}^{T} & \mathbf{A}_{4}^{T} & \mathbf{A}_{5}^{T} & \mathbf{A}_{6}^{T} & \mathbf{A}_{7}^{T} & \mathbf{A}_{8}^{T}\end{array}\right]^{T}$ be the right nullspace of matrix $\mathbf{B}$. Hereafter, we employ the relation $\mathbf{B A}=\mathbf{0}$ to determine the elements of $\mathbf{A}$. Specifically, from the second, third, fourth and seventh rows of the product $\mathbf{B A}$, we have:

$$
\mathbf{A}_{5}=\mathbf{A}_{6}=\mathbf{A}_{7}=\mathbf{A}_{8}=\mathbf{0}
$$

Then, from the sixth row of the product, we have the relation $\lfloor\mathbf{C g}\rfloor \frac{\partial \boldsymbol{\theta}}{\partial \mathbf{s}} \mathbf{A}_{1}=\mathbf{0}$, thus $\mathbf{A}_{\mathbf{1}}=\frac{\partial \mathbf{s}}{\partial \boldsymbol{\theta}} \mathbf{C g}$ or $\mathbf{A}_{\mathbf{1}}=\mathbf{0}$.

If $\mathbf{A}_{\mathbf{1}}=\frac{\partial \mathbf{s}}{\partial \boldsymbol{\theta}} \mathbf{C g}$, from the fifth row of $\mathbf{B A}$ we have $\mathbf{A}_{\mathbf{2}}=$ $-\lfloor\mathbf{v}\rfloor \mathbf{g}$, and from the first row, we have $\mathbf{A}_{\mathbf{3}}=-\lfloor\mathbf{p}\rfloor \mathbf{g}$ and $\mathbf{A}_{4}=-\left\lfloor\mathbf{p}_{f}\right\rfloor \mathbf{g}$.

If $\mathbf{A}_{\mathbf{1}}=\mathbf{0}$, from the fifth row of $\mathbf{B A}$ we have $\mathbf{A}_{\mathbf{2}}=\mathbf{0}$, and from the first row $\mathbf{A}_{\mathbf{3}}=\mathbf{A}_{\mathbf{4}}=\mathbf{I}$.

Using Gaussian elimination, it is easy to show that the rank of matrix $\mathbf{B}$ is 20 , thus the dimension of its right nullspace is exactly four as we have found. In summary, the system's unobservable directions are spanned by the matrix:

$$
\mathbf{N} \triangleq\left[\begin{array}{cc}
\frac{\partial \mathbf{s}}{\partial \boldsymbol{\theta}} \mathbf{C g} & \mathbf{0} \\
-\lfloor\mathbf{v}\rfloor \mathbf{g} & \mathbf{0} \\
-\lfloor\mathbf{p}\rfloor \mathbf{g} & \mathbf{I}_{3} \\
-\left\lfloor\mathbf{p}_{f}\right\rfloor \mathbf{g} & \mathbf{I}_{3} \\
\mathbf{0} & \mathbf{0} \\
\mathbf{0} & \mathbf{0} \\
\mathbf{0} & \mathbf{0} \\
\mathbf{0} & \mathbf{0}
\end{array}\right]=\left[\begin{array}{ll}
\mathbf{N}^{g} & \mathbf{N}^{p}
\end{array}\right]
$$

where the first row is the perturbing term with respect to $\mathbf{s}$, while the corresponding term for $\boldsymbol{\theta}$ is $\mathbf{C g}$. Therefore, the unobservable directions are the global position of the IMURGBD camera and the point landmark, $\mathbf{N}^{p}$, and their rotation about the gravity vector, $\mathbf{N}^{g}$.

\section{Algorithm DESCRIPTION}

We implemented the IMU-RGBD camera 3D pose estimation and extrinsic calibration system (16) with the OCEKF proposed in [13]. Specifically, in [13] it was shown that due to linearization errors, the filter gains spurious information along unobservable directions, which results in smaller uncertainty (that causes the filter to be inconsistent) and larger estimation errors. To address this problem, the authors proposed to modify the Jacobian matrices in both the OC-EKF's propagation and update steps, so as to avoid changing the unobservable directions due to the linearization errors.

Hereafter, we briefly describe the methodology of the OC-EKF, while the interested reader is referred to [13] for details. In OC-EKF, the spurious information is removed by enforcing the following observability constraints:

$$
\begin{gathered}
\mathbf{N}_{k+1}=\boldsymbol{\Phi}_{k} \mathbf{N}_{k} \\
\mathbf{H}_{k} \mathbf{N}_{k}=\mathbf{0}, \quad \forall k>0
\end{gathered}
$$

where $\mathbf{N}_{k}$ and $\mathbf{N}_{k+1}$ are the unobservable directions presented in (38) at time-steps $k$ and $k+1$, respectively, $\boldsymbol{\Phi}_{k}$ is the Jacobian matrix of the system propagation equation (14) at time step $k$ and $\mathbf{H}_{k}$ is the Jacobian matrix of the system measurement equation (15) at time step $k$.

In [13], the authors showed that the two constraints (39) and (40) are both satisfied when the EKF is linearized with the true state $\mathbf{x}$. However, when linearized with the estimated state $\hat{\mathbf{x}}$, as in practice, neither (39) nor (40) hold. To address this problem, hereafter we will show how to appropriately modify $\boldsymbol{\Phi}_{k}$ and $\mathbf{H}_{k}$ so as to satisfy the two constraints thus retain the system's observability properties.

(1) Modification of the State Transition Matrix $\boldsymbol{\Phi}_{k}$ : Substituting the nullspace defined in (38), the observability constraint (39) can be split into two constraints:

$$
\begin{aligned}
& \mathbf{N}_{k+1}^{p}=\boldsymbol{\Phi}_{k} \mathbf{N}_{k}^{p} \\
& \mathbf{N}_{k+1}^{g}=\boldsymbol{\Phi}_{k} \mathbf{N}_{k}^{g}
\end{aligned}
$$

Due to the structure of $\boldsymbol{\Phi}_{k}$, as shown in [13], the constraint (41) is automatically satisfied because all the block rows result in $\mathbf{0}_{3}=\mathbf{0}_{3}$ or $\mathbf{I}_{3}=\mathbf{I}_{3}$. However, to fulfill constraint (42), we need to modify $\boldsymbol{\Phi}_{k}$ so as to satisfy the following three constraints:

$$
\begin{gathered}
\boldsymbol{\Phi}_{11} \mathbf{C}_{k} \mathbf{g}=\mathbf{C}_{k+1} \mathbf{g} \\
\boldsymbol{\Phi}_{21} \mathbf{C}_{k} \mathbf{g}=\left\lfloor\mathbf{v}_{k}\right\rfloor \mathbf{g}-\left\lfloor\mathbf{v}_{k+1}\right\rfloor \mathbf{g} \\
\boldsymbol{\Phi}_{31} \mathbf{C}_{k} \mathbf{g}=\delta t\left\lfloor\mathbf{v}_{k}\right\rfloor \mathbf{g}+\left\lfloor\mathbf{p}_{k}\right\rfloor \mathbf{g}-\left\lfloor\mathbf{p}_{k+1}\right\rfloor \mathbf{g}
\end{gathered}
$$

where $\boldsymbol{\Phi}_{11}, \boldsymbol{\Phi}_{21}, \boldsymbol{\Phi}_{31}$ are $3 \times 3$ matrices in the first block column of the first, second and third block rows of state transition matrix $\boldsymbol{\Phi}_{k}$. Constraint (43) can be simply satisfied by modifying $\boldsymbol{\Phi}_{11}^{*}=\mathbf{C}_{k+1} \mathbf{C}_{k}^{T}$.

Both of (44) and (45) are in the form $\mathbf{A u}=\mathbf{w}$, where $\mathbf{u}$ and $\mathbf{w}$ comprise nullspace elements that are fixed, and we seek to select another matrix $\mathbf{A}^{*}$ that is closest to the original matrix $\mathbf{A}$ in the Frobenius norm sense, while satisfying constraints (44) and (45). To do so, we formulate the following optimization problem

$$
\begin{gathered}
\mathbf{A}^{*}=\underset{\mathbf{A}^{*}}{\operatorname{argmin}}\left\|\mathbf{A}^{*}-\mathbf{A}\right\|_{\mathcal{F}}^{2} \\
\text { s. t. } \quad \mathbf{A}^{*} \mathbf{u}=\mathbf{w}
\end{gathered}
$$

where $\|\cdot\|_{\mathcal{F}}$ denotes the Frobenius matrix norm. The optimal $\mathbf{A}^{*}$ can be determined by solving its KKT optimality condition [18], whose solution is

$$
\mathbf{A}^{*}=\mathbf{A}-(\mathbf{A} \mathbf{u}-\mathbf{w})\left(\mathbf{u}^{T} \mathbf{u}\right)^{-1} \mathbf{u}^{T}
$$

(2) Modification of the Measurement Jacobian $\mathbf{H}_{k}$ : During the update, we seek to modify the Jacobian matrix $\mathbf{H}_{k}$ so as 
to fulfill constraint (40), i.e.,

$$
\begin{aligned}
\mathbf{H}_{k} \mathbf{N}_{k}^{p} & =0 \\
\mathbf{H}_{k} \mathbf{N}_{k}^{g} & =0
\end{aligned}
$$

where

$$
\mathbf{H}_{k}=\left[\begin{array}{lllllll}
\mathbf{H}_{\boldsymbol{\theta}} & \mathbf{0}_{3 \times 3} & \mathbf{H}_{\mathbf{p}} & \mathbf{H}_{\mathbf{p}_{f}} & \mathbf{0}_{3 \times 6} & \mathbf{H}_{\boldsymbol{\theta}_{C}} & \mathbf{H}_{\mathbf{p}_{C}}
\end{array}\right]
$$

in which $\mathbf{H}_{\boldsymbol{\theta}}, \mathbf{H}_{\mathbf{p}}, \mathbf{H}_{\mathbf{p}_{f}}, \mathbf{H}_{\boldsymbol{\theta}_{C}}$ and $\mathbf{H}_{\mathbf{p}_{C}}$ are the Jacobian matrices corresponding to $\boldsymbol{\theta}, \mathbf{p}, \mathbf{p}_{f}, \boldsymbol{\theta}_{C}$ and $\mathbf{p}_{C}$. The only nonzero elements in $\mathbf{N}_{k}^{p}$ are identity matrices corresponding to the landmark and sensor position. Therefore, constraint (48) requires $\mathbf{H}_{f}=-\mathbf{H}_{p}$.

On the other hand, constraint (49) requires

$$
\left[\begin{array}{ll}
\mathbf{H}_{\boldsymbol{\theta}} & \mathbf{H}_{p}
\end{array}\right]\left[\begin{array}{c}
\mathbf{C}_{k} \mathbf{g} \\
\left(\left\lfloor\mathbf{p}_{f}\right\rfloor-\left\lfloor\mathbf{p}_{k}\right\rfloor\right) \mathbf{g}
\end{array}\right]=\mathbf{0}
$$

This is a constraint of the form $\mathbf{A u}=\mathbf{0}$, where $\mathbf{u}$ is a fixed quantity determined by elements in the nullspace, and A comprises elements of the measurement Jacobian $\mathbf{H}_{k}$. We compute the optimal $\mathbf{A}^{*}$ that satisfies this relationship using (46) and (47), for the special case when $\mathbf{w}=\mathbf{0}$.

\section{Simulation AND EXPERIMENTAl RESUlts}

We have employed the OC-EKF in both our simulations and experiments, to: (i) Demonstrate the accuracy of our proposed IMU-RGBD camera 3D pose estimation and extrinsic calibration algorithm; (ii) Show that by removing spurious information, the OC-EKF outperforms the standard EKF; and (iii) Verify the system's unobservable directions by demonstrating the improvement of the OC-EKF which was designed using these unobservable directions.

\section{A. Simulation Results}

In our simulation, the RGBD camera observes 20 landmarks, which are randomly generated in a plane. The IMU travels in front of this plane with constant local rotational velocity $\left[\begin{array}{lll}2.8648 & 0 & 2.8648\end{array}\right]^{T} \mathrm{deg} / \mathrm{sec}$ and linear acceleration $\left[\begin{array}{lll}0 & 0.1 & 0\end{array}\right]^{T} \mathrm{~m} / \mathrm{sec}^{2}$. The initial IMU velocity is set to $\left[\begin{array}{lll}0.02 & 0 & 0.01\end{array}\right]^{T} \mathrm{~m} / \mathrm{sec}$. The rotation matrix and translation of the IMU in the RGBD camera frame is set to $\left[\begin{array}{lllllllll}-1 & 0 & 0 ; & 0 & 0 & 1 ; & 0 & 1 & 0\end{array}\right]$ and $\left[\begin{array}{lll}.1 & .1 & .1\end{array}\right]^{T} \mathrm{~m}$. The initial standard deviation of the translation and rotation is set to $0.02 \mathrm{~m}$ and 2 degrees.

First, we examine the performance of the OC-EKF and EKF in a single run. The estimation errors and $3 \sigma$ bounds of the IMU's orientation around the gravity vector are shown in Fig. 1(a). As evident, the EKF gains spurious information, (its covariance decreases over time), while the covariance of the OC-EKF does not. In addition, the EKF becomes inconsistent gradually, while the OC-EKF remains consistent.

In Fig. 1(b) and Fig. 1(c), we present the results of 20 Monte Carlo simulations. The RMSE (root mean square errors) for the IMU position, orientation, and the transformation between the two sensors are all smaller for the OC$\mathrm{EKF}$ as compared to the EKF, which verifies the validity of our observability analysis based on which the OC-EKF was designed.

\section{B. Experimental Results}

We compare the performance of the OC-EKF with the EKF in practice when performing simultaneous localization, mapping and extrinsic IMU-RGBD camera calibration. In our experimental setup, we utilize a platform comprised of an InterSense NavChip IMU and a kinect, which has both an RGB camera and an infrared (IR) depth-finding camera. The intrinsic parameters of the kinect RGB camera and IR camera, as well as the transformation between the two cameras, are calibrated offline with the algorithm described in [5].

We train a vocabulary tree [19] with the SIFT features extracted in an indoor environment. During the experiments, the SIFT features of each image are passed through into the vocabulary tree's leaves, and the leave indexes for each image are recorded. Once a new image is captured, we pass all its SIFT features through the vocabulary tree to its leaves, and find the images with the most similar SIFT features. Then the identities of the SIFT features in the current image are determined by matching them to SIFT features in similar images and employing a geometry consistency test. Since the transformation between the IR camera and the RGB camera is known, the corresponding 3D points captured by the IR camera can also be associated.

In our experiment, the IMU-kinect platform travels in an indoor office along a closed-loop trajectory, which means at the end the platform comes back to its starting location. This information is used to assess the estimated robot position accuracy. The experimental results are depicted in Fig. 2. The black line is the platform's trajectory and the red stars denote the estimated landmarks. The whole trajectory is 7.04 $\mathrm{m}$, and the final position error is $7.5 \mathrm{~cm}$ for the OC-EKF and $8.1 \mathrm{~cm}$ for the EKF. We also plot the estimated translation and rotation between the IMU and kinect in Fig. 2. As evident, the estimated transformation when using the OC$\mathrm{EKF}$ is more stable than that from the EKF.

\section{CONCLUSION}

In this paper, we present an observability analysis for the IMU-RGBD camera 3D pose estimation and extrinsic calibration problem in an unknown environment using Lie derivatives. Due to the requirement of finding the infinite dimensional observability matrix's nullspace, it is quite challenging to determine a system's unobservable directions. To address this problem, we decompose the observability matrix into the product of two matrices, $\Xi$ and $\mathbf{B}$, using a nonlinear transformation (basis functions) of the original variables. Then we find the system's unobservable directions from the nullspace of matrix $\mathbf{B}$, which has finite dimensions. Moreover, we construct an observable system using the basis functions whose observability matrix is matrix $\Xi$, and show that the basis functions are the IMU-RGBD camera system's observable modes. We present extensive simulation 


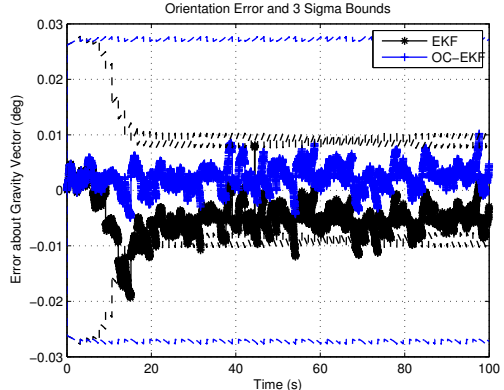

(a)
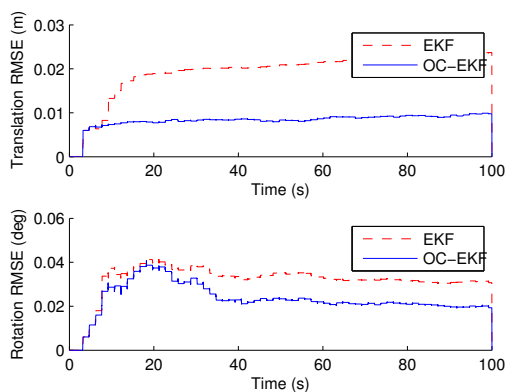

(b)
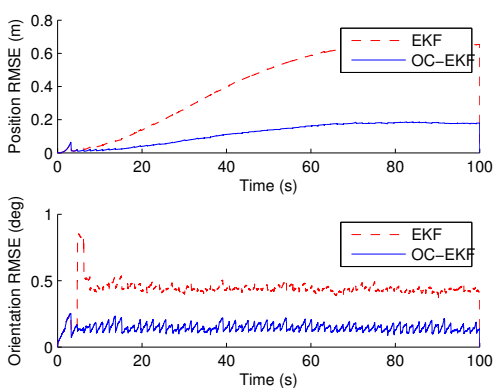

(c)

Fig. 1. Simulation results for the OC-EKF and EKF: (a) Error and $3 \sigma$ bounds for the rotation about the gravity vector in a single run. (b) RMSE for the translation and rotation between the two sensors over 20 Monte Carlo trials. (c) RMSE for the robot position and orientation over 20 Monte Carlo trials.

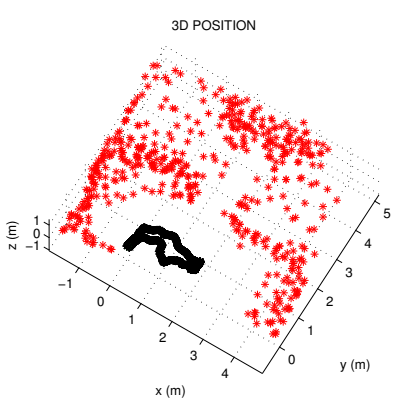

(a)

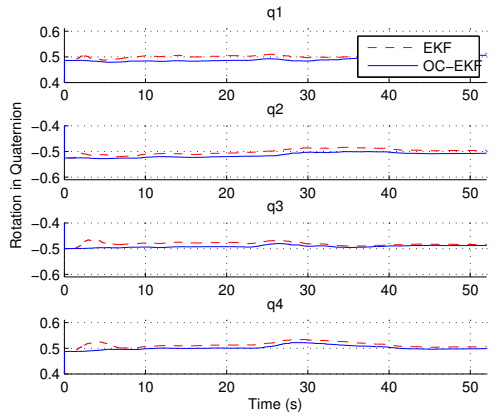

(b)

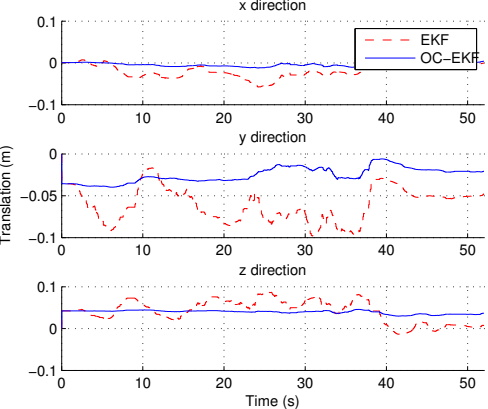

(c)

Fig. 2. Experimental results: (a) The platform trajectory and estimated landmarks. (b) The rotation between the IMU and the kinect in quaternion representation. (c) The translation between the IMU and the kinect.

and experimental results to show that the observabilityconstrained (OC)-EKF designed to adhere to the system's unobservable directions significantly outperforms the EKF.

In our future work, we plan to perform observability analysis of the IMU-camera extrinsic calibration system employing the same methodology and improve its consistency.

\section{REFERENCES}

[1] A. I. Mourikis, N. Trawny, S. I. Roumeliotis, A. E. Johson, A. Ansar, and L. Matthies, "Vision-aided inertial navigation for spacecraft entry, descent, and landing," IEEE Trans. on Robotics, vol. 25, no. 2, pp. 264-280, Apr. 2009.

[2] [Online]. kinectforwindows/

[3] R. O. Allen and D. H. Change, "Performance testing of the systron donner quartz gyro (qrs11-100-420); sn's 3332, 3347 and 3544,” JPL, Tech. Rep., 1993.

[4] J. Y. Bouguet, "Camera calibration toolbox for matlab," 2006. [Online]. Available: http://www.vision.caltech.edu/bouguetj/calibdoc/

[5] D. Herrera, J. Kannala, and J. Heikkila, "Joint depth and color camera calibration with distortion correction," IEEE Trans. on Pattern Analysis and Machine Intelligence, vol. 34, no. 10, pp. 2058-2064, Oct. 2012.

[6] P. Lang and A. Pinz, "Calibration of hybrid vision/inertial tracking systems," in Proc. of the 2nd InerVis: Workshop on Integration of Vision and Inertial Sensors, Barcelona, Spain, Apr. 182005.

[7] J. Lobo and J. Dias, "Relative pose calibration between visual and inertial sensors," International Journal of Robotics Research, vol. 26, no. 6, pp. 561-575, June 2007.

[8] F. M. Mirzaei and S. I. Roumeliotis, "A kalman filter-based algorithm for imu-camera calibration: Observability analysis and performance evaluation," IEEE Trans. on Robotics, vol. 24, no. 5, pp. 1143-1156, Oct. 2008
[9] J. Kelly and G. S. Sukhatme, "Visual-inertial sensor fusion: Localization, mapping and sensor-to-sensor self-calibration," International Journal of Robotics Research, vol. 30, no. 1, pp. 56-79, Jan. 2011.

[10] S. Weiss, M. W. Achtelik, S. Lynen, M. Chli, and R. Siegwart, "Realtime onboard visual-inertial state estimation and self-calibration of mavs in unknown environments," in Proc. of the IEEE International Conference on Robotics and Automation, Saint Paul, Minnesota, May 14-18 2012, pp. 957-964.

[11] J. A. Hesch, "Toward consistent vision-aided inertial navigation," Ph.D. dissertation, University of Minnesota, 2013.

[12] R. Hermann and A. J. Krener, "Nonlinear controllability and observability," IEEE Trans. on Automatic Control, vol. 22, no. 5, pp. 728740, Oct. 1977.

[13] J. A. Hesch, D. G. Kottas, S. L. Bowman, and S. I. Roumeliotis, "Observability-constrained vision-aided inertial navigation," University of Minnesota, Dept. of Comp. Sci. \& Eng., MARS Lab, Tech. Rep., Feb. 2012.

[14] A. Martinelli, "State estimation based on the concept of continuous symmetry and observability analysis: The case of calibration," IEEE Trans. on Robotics, vol. 27, no. 2, pp. 239-255, Apr. 2011.

[15] C. X. Guo and S. I. Roumeliotis, "Imu-rgbd camera extrinsic calibration in unknown environments: Observability analysis and consistency improvement," University of Minnesota, Tech. Rep., 2012. [Online]. Available: http://www-users.cs.umn.edu/ chaguo/

[16] M. D. Shuster, "A survey of attitude representations," the Journal of Astronautical Sciences, vol. 41, no. 4, pp. 439-517, Oct.-Dec. 1993.

[17] H. H. Chen, "Pose determination from line-to-plane correspondences: existence condition and closed-form solutions," IEEE Trans. on Pattern Analysis and Machine Intelligence, vol. 13, no. 6, pp. 530-541, June 1991.

[18] S. Boyd and L. Vandenberghe, Convex Optimization. Cambridge University Press, 2004.

[19] D. Nister and H. Stewenius, "Scalable recognition with a vocabulary tree," in Proc. of the IEEE Conference on Computer Vision and Pattern Recognition, New York, NY, June 2006, pp. 2161-2168. 